\title{
High-Order Neural Networks and Kernel Methods for Peptide-MHC Binding Prediction
}

Pavel P. Kuksa, Martin Renqiang Min*, Rishabh Dugar, and Mark Gerstein*

* To whom correspondence should be addressed

\section{Supplementary Information}

\section{Model hyperparameters}

We set the hyperparameters for both DNN and HONN by optimizing their performance on the validation set (20\% of the training data) for each target allele. The found sets of parameters were used for training on the full train dataset(IEDB) and making predictions for the corresponding test data of each target allele. The selected set of hyperparameters is shown in Table S1.

Table S1: DNN and HONN hyperparameters used for training and pre-training

\begin{tabular}{c|c|ccc}
\hline Model & param & A0201 & A0206 & A2402 \\
\hline \hline \multirow{7}{*}{ DNN } & \# hidden units $h_{1}$ (layer 1) & 16 & 32 & 16 \\
& \# hidden units $h_{2}$ (layer 2) & 16 & 16 & 16 \\
& \# hidden units $h_{3}$ (layer 3) & 4 & 4 & 8 \\
& fine-tuning learning rate & 0.008 & 0.008 & 0.008 \\
& fine-tuning batch size & 1 & 1 & 1 \\
& fine-tuning epochs & 30 & 60 & 30 \\
& pre-training learning rate & 0.005 & 0.05 & 0.05 \\
& pre-training batch size & 100 & 100 & 100 \\
& pre-training epochs & 30 & 10 & 30 \\
\hline \multirow{6}{*}{ HONN } & \# mean hidden units $h^{m}$ & 4 & 8 & 8 \\
& \# high-order (covariance) units $h^{g}$ & 4 & 4 & 4 \\
& \# factors & 8 & 8 & 8 \\
& fine-tuning learning rate & 0.0015 & 0.0011 & 0.005 \\
& fine-tuning batch size & 1 & 1 & 1 \\
& fine-tuning epochs & 30 & 30 & 30 \\
& pre-training learning rate & 0.05 & 0.05 & 0.05 \\
& pre-training batch size & 100 & 100 & 100 \\
& pre-training epochs & 20 & 20 & 20 \\
\hline
\end{tabular}




\section{Comparison of the performance for pre-trained models vs models without pre-training}

Tables S2, S4, S6 show the quality of ranking (nDCG) MHC-peptide interactions on test data using DNN trained with or without pre-training. Similarly, Tables S3, S5, S7 show the quality of ranking MHC-peptide interactions using high-order neural network (HONN) trained with or without pre-training. Pre-training of the models consistently improves their performance.

Table S2: A0201-Japanese data. Relevance/ranking assessment (nDCG)

\begin{tabular}{lccccc}
\hline method & nDCG@10 & nDCG@20 & nDCG@30 & nDCG@50 & nDCG \\
\hline \hline DNN (no pre-training) & 47.26 & 54.36 & 61.56 & 67.98 & 81.32 \\
DNN (with pre-training) & 63.89 & 65.59 & 70.12 & 74.57 & 86.33 \\
\hline
\end{tabular}

Table S3: A0201-Japanese data. Relevance/ranking assessment (nDCG)

\begin{tabular}{lccccc}
\hline method & nDCG@10 & nDCG@20 & nDCG@30 & nDCG@50 & nDCG \\
\hline \hline HONN (no pre-training) & 63.07 & 64.30 & 66.81 & 74.54 & 85.54 \\
HONN (with pre-training) & 63.93 & 65.94 & 70.61 & 75.55 & 86.46 \\
\hline
\end{tabular}

Table S4: A0206-Japanese data. Relevance/ranking assessment (nDCG)

\begin{tabular}{lcccc}
\hline method & nDCG@10 & nDCG@20 & nDCG@30 & nDCG \\
\hline \hline DNN (no pre-training) & 75.83 & 79.30 & 79.03 & 89.81 \\
DNN (with pre-training) & 77.50 & 82.21 & 81.72 & 91.74 \\
\hline
\end{tabular}

Table S5: A0206-Japanese data. Relevance/ranking assessment (nDCG)

\begin{tabular}{lcccc}
\hline method & nDCG@10 & nDCG@20 & nDCG@30 & nDCG \\
\hline \hline HONN (no pre-training) & 74.14 & 77.86 & 76.90 & 88.72 \\
HONN (with pre-training) & 75.39 & 78.06 & 79.92 & 90.80 \\
\hline
\end{tabular}

Table S6: A2402-Japanese data. Relevance/ranking assessment (nDCG)

\begin{tabular}{lcccc}
\hline method & nDCG@10 & nDCG@20 & nDCG@30 & nDCG \\
\hline \hline DNN (no pre-training) & 48.49 & 51.22 & 55.11 & 82.21 \\
DNN (with pre-training) & 51.07 & 53.78 & 56.88 & 84.36 \\
\hline
\end{tabular}


Table S7: A2402-Japanese data. Relevance/ranking assessment (nDCG)

\begin{tabular}{lccccc}
\hline method & nDCG@5 & nDCG@10 & nDCG@20 & nDCG@30 & nDCG \\
\hline \hline HONN (no pre-training) & 52.17 & 50.38 & 56.83 & 60.60 & 84.96 \\
HONN (with pre-training) & 55.73 & 57.36 & 57.20 & 60.82 & 85.20 \\
\hline
\end{tabular}

\section{Evaluation on benchmark IEDB data}

To further evaluate performance of proposed methods, we used recent benchmark MHC-I binding datasets from (Kim et al. 2014 ). We first trained a deep neural network (DNN), a proposed high-order semi-RBM (HONN), and a highorder kernel SVM (hkSVM) on BD2009 binding datasets (Kim et al., 2014) for each allele. We then tested on the independent blind dataset (BLIND) for each MHC-I allele. Table S8 shows training (BD2009) and independent test (BLIND) datasets used for evaluation.

To train HONN, we first select hyper-parameters (number of hidden units and learning rate) on the validation set (20\% of the training BD2009 allele data). Selected hyper-parameters are then used to train on the full training dataset. Trained models are then tested on the independent BLIND test sets for each allele.

Table S9 shows AUC and ROC- $n$ scores for the high-order kernel SVM (hkSVM), high-order semi-RBM (HONN), and NetMHC method (Nielsen et al. 2003: Buus et al., 2003; Lundegaard et al., 2011). As can be seen from the results in the table, while the area under ROC curve (AUC) scores are very similar for both our method and the NetMHC method (AUC scores of 92.875 and 92.375), for the very highest ranked peptides (low false positive (FP) rates), both hkSVM and HONN+hkSVM perform better on average compared to NetMHC as measured by ROC- $n$ scores (e.g., ROC-1 scores of hkSVM or HONN are higher in about $67 \%(31 / 46)$ of the tested alleles and tied in $22 \%(10 / 46)$ of the tested alleles).

Peptide binding prediction scores on the test (BLIND) datasets for each allele are provided in the supplementary data (hksvm_honn_blind_predict.tar.gz). 
Table S8: MHC-I BD2009 (train), MHC-I BLIND (test) benchmark datasets (Kim et al. 2014) (human MHC-I alleles)

Train \#peptides \#binders \#non-binders $\mid$ Test \#peptides \#binders \#non-binders

\begin{tabular}{|c|c|c|c|c|c|c|c|}
\hline$\overline{\mathrm{A} \text { A0201-BD2009 }}$ & 26759 & 2070 & 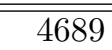 & A0201-BLIND & $\overline{17778}$ & 935 & 8843 \\
\hline A2601-BD2009 & 2337 & 247 & 2090 & A2601-BLIND & 1132 & 86 & 1046 \\
\hline B0801-BD2009 & 2068 & 459 & 1609 & B0801-BLIND & 707 & 191 & 516 \\
\hline B5101-BD2009 & 1294 & 162 & 1132 & B5101-BLIND & 673 & 46 & 627 \\
\hline B5701-BD2009 & 1649 & 207 & 1442 & B5701-BLIND & 647 & 145 & 502 \\
\hline B3901-BD2009 & 876 & 168 & 708 & B3901-BLIND & 641 & 25 & 616 \\
\hline B1501-BD2009 & 3114 & 828 & 2286 & B1501-BLIND & 633 & 215 & 418 \\
\hline A0301-BD2009 & 4533 & 944 & 3589 & A0301-BLIND & 591 & 330 & 261 \\
\hline B0702-BD2009 & 2914 & 539 & 2375 & B0702-BLIND & 589 & 365 & 224 \\
\hline B1517-BD2009 & 841 & 267 & 574 & B1517-BLIND & 582 & 93 & 489 \\
\hline A3101-BD2009 & 3266 & 676 & 2590 & A3101-BLIND & 521 & 263 & 258 \\
\hline A1101-BD2009 & 3798 & 1119 & 2679 & A1101-BLIND & 514 & 193 & 321 \\
\hline B0802-BD2009 & 486 & 19 & 467 & B0802-BLIND & 509 & 18 & 491 \\
\hline B1801-BD2009 & 1626 & 181 & 1445 & B1801-BLIND & 503 & 28 & 475 \\
\hline A0206-BD2009 & 3122 & 1211 & 1911 & A0206-BLIND & 482 & 251 & 231 \\
\hline A0101-BD2009 & 3102 & 383 & 2719 & A0101-BLIND & 479 & 77 & 402 \\
\hline A3001-BD2009 & 1933 & 567 & 1366 & A3001-BLIND & 470 & 151 & 319 \\
\hline A6901-BD2009 & 2073 & 220 & 1853 & A6901-BLIND & 470 & 26 & 444 \\
\hline B1509-BD2009 & 345 & 16 & 329 & B1509-BLIND & 466 & 29 & 437 \\
\hline A0203-BD2009 & 3822 & 1205 & 2617 & A0203-BLIND & 460 & 255 & 205 \\
\hline A6802-BD2009 & 3085 & 607 & 2478 & A6802-BLIND & 457 & 188 & 269 \\
\hline B2703-BD2009 & 433 & 0 & 433 & B2703-BLIND & 441 & 0 & 441 \\
\hline A2501-BD2009 & 519 & 66 & 453 & A2501-BLIND & 416 & 5 & 411 \\
\hline A2602-BD2009 & 202 & 67 & 135 & A2602-BLIND & 413 & 99 & 314 \\
\hline A8001-BD2009 & 774 & 111 & 663 & A8001-BLIND & 379 & 7 & 372 \\
\hline B4601-BD2009 & 1355 & 77 & 1278 & B4601-BLIND & 378 & 0 & 378 \\
\hline B3801-BD2009 & 136 & 3 & 133 & B3801-BLIND & 351 & 141 & 210 \\
\hline A2402-BD2009 & 1904 & 316 & 1588 & A2402-BLIND & 346 & 136 & 210 \\
\hline B3501-BD2009 & 1945 & 478 & 1467 & B3501-BLIND & 341 & 162 & 179 \\
\hline A2603-BD2009 & 205 & 25 & 180 & A2603-BLIND & 312 & 37 & 275 \\
\hline A6801-BD2009 & 1665 & 629 & 1036 & A6801-BLIND & 312 & 186 & 126 \\
\hline B5301-BD2009 & 576 & 190 & 386 & B5301-BLIND & 296 & 120 & 176 \\
\hline A3301-BD2009 & 1579 & 223 & 1356 & A3301-BLIND & 288 & 161 & 127 \\
\hline B5801-BD2009 & 2371 & 367 & 2004 & B5801-BLIND & 275 & 158 & 117 \\
\hline A3002-BD2009 & 867 & 230 & 637 & A3002-BLIND & 267 & 103 & 164 \\
\hline A3201-BD2009 & 569 & 275 & 294 & A3201-BLIND & 256 & 120 & 136 \\
\hline B4402-BD2009 & 1262 & 101 & 1161 & B4402-BLIND & 250 & 129 & 121 \\
\hline B2705-BD2009 & 2294 & 335 & 1959 & B2705-BLIND & 249 & 26 & 223 \\
\hline B4001-BD2009 & 2288 & 274 & 2014 & B4001-BLIND & 243 & 100 & 143 \\
\hline A2301-BD2009 & 1429 & 269 & 1160 & A2301-BLIND & 242 & 86 & 156 \\
\hline B0803-BD2009 & 217 & 9 & 208 & B0803-BLIND & 234 & 9 & 225 \\
\hline B4403-BD2009 & 474 & 96 & 378 & B4403-BLIND & 205 & 103 & 102 \\
\hline B1503-BD2009 & 404 & 331 & 73 & B1503-BLIND & 165 & 33 & 132 \\
\hline A0202-BD2009 & 2212 & 1003 & 1209 & A0202-BLIND & 126 & 44 & 82 \\
\hline A2902-BD2009 & 1736 & 427 & ${ }^{4} 1309$ & A2902-BLIND & 118 & 27 & 91 \\
\hline B5401-BD2009 & 577 & 127 & 450 & B5401-BLIND & 79 & 5 & 74 \\
\hline B4002-BD2009 & 450 & 154 & 296 & B4002-BLIND & 74 & 18 & 56 \\
\hline B4501-BD2009 & 459 & 101 & 358 & B4501-BLIND & 65 & 5 & 60 \\
\hline
\end{tabular}


Table S9: Comparison of AUC scores on independent (BLIND) dataset for human MHC-I alleles. Proposed methods display state-of-the-art prediction performance when tested across 46 human MHC-I alleles. Observed improvements in ROC- $n$ scores (low FP rates) are significant (paired signed rank test P-values=7e-3 and 1.38e-2 for hkSVM and HONN+hkSVM, respectively).

\begin{tabular}{|c|c|c|c|c|c|c|c|c|c|c|c|c|c|c|c|c|}
\hline \multirow{2}{*}{$\begin{array}{l}\text { Allele } \\
\text { 01-BLIND }\end{array}$} & \multirow{2}{*}{$\frac{\mathrm{AUC}}{\mathrm{92.84}}$} & $\begin{array}{r}\text { Netl } \\
\text { ROC-1 }\end{array}$ & NetMHC & $\mathrm{R}$ & \multicolumn{4}{|c|}{ hkSVM } & \multicolumn{4}{|c|}{ HONN } & \multicolumn{3}{|c|}{ HONN+hkSVM } & $\mathrm{R}$ \\
\hline & & .09 & 26.23 & & $\overline{8.04}$ & $\overline{99}$ & 17.14 & 77 & 3.60 & & 27.7 & & . & 16.88 & 18.96 & \\
\hline & & 2.57 & 9.09 & & & 7 & & & & & & & & 3.97 & 9.05 & \\
\hline & & 50.00 & 60.45 & & & 61.36 & 66.82 & & & 43.18 & 61.36 & & & 56.82 & 65.45 & \\
\hline & & 67.06 & 79.22 & & & 69.02 & 81.57 & & & 38.82 & 70.98 & & & 67.84 & 81.49 & \\
\hline & & 13.15 & 44.22 & & & 20.72 & 48.84 & & & 26.69 & 35.22 & & & 20.72 & 48.53 & \\
\hline & & 4.85 & 11.39 & & & 9.39 & 17.52 & & & 8.48 & 23.15 & & & 8.79 & 17.45 & \\
\hline & & 9.33 & 41.66 & 52 & 77 & 13.99 & 38.55 & & & 25.91 & 35.85 & & 79 & 13.47 & 39.27 & \\
\hline & & 8.14 & 26.98 & & & & & & & & & & & 18.60 & 24.42 & \\
\hline & & 6.62 & 15.59 & & & & 18.82 & & & & & & & 13.97 & 19.56 & \\
\hline & & 40.00 & 72.00 & & & & 52.00 & & & & 60.00 & & & 40.00 & 52.00 & \\
\hline & & 16.28 & 24.42 & & & 20.93 & 25.81 & & & 15.12 & 19.30 & & & 18.60 & 24.88 & \\
\hline & & 20.20 & 41.21 & & & 33 & 49.70 & & & 24.24 & 29.29 & & 27 & 31.31 & 50.91 & \\
\hline & & 21.62 & 35.68 & & & & 43.78 & & & 2.70 & & & & 27.03 & 42.16 & \\
\hline & & 59.26 & 59.26 & & & 26 & 62.22 & & & 40.74 & 60 & & & 59.26 & 62.96 & \\
\hline & & 5.30 & 12.32 & & & & 15.23 & & & & 29.27 & & & 7.28 & 14.97 & \\
\hline & & 0.99 & 4.36 & & & 7 & 8.51 & & & 3.96 & & & 97 & 2.97 & 7.92 & \\
\hline & & 2.28 & 14.75 & & & & 7.26 & & & 9.51 & & & & 8.37 & .26 & \\
\hline & & 67 & 14.67 & & & 83 & 19. & & & 6.67 & & & & 10.83 & & \\
\hline & & 0.00 & 19.13 & & & & 34.16 & & & & & & & 6.21 & 33.91 & \\
\hline & & .73 & 48.17 & & & 2 & 3 & & & 50 & & & & 34 & 77 & \\
\hline & & .91 & 82.98 & & & 26 & 69.15 & & & & 62 & & & 32 & 47 & \\
\hline & & 1.62 & 41.54 & & & & 41.54 & & & & & & & & & \\
\hline & & .57 & 51.43 & & & & & & & & & & & & & \\
\hline & & .55 & 27.29 & & & 28.22 & 32.60 & & & 22.47 & & & & 59 & 32.27 & \\
\hline & & 23.04 & 27.02 & & & 24.08 & 28.06 & & & & & & & 4.08 & 27.75 & \\
\hline & & 5.56 & 7.78 & & & & 40.00 & & & & & & & & 42.22 & \\
\hline & & 00 & 13.33 & & & & 20 & & & & & & & & & \\
\hline & & .16 & 20.84 & & & & & & & & & & & 12.09 & 23.07 & \\
\hline & 8 & 1.21 & 26.67 & & & 8 & 23.64 & & & & & & & 18.18 & 23.64 & \\
\hline & & 17.24 & 24.14 & & & & & & & & & & & & & \\
\hline & & 24.73 & 36.34 & & & & 34 & & & & & & 71 & 27.96 & 33.98 & \\
\hline & & & 19.29 & & & & & & & & & & & 57 & 9.29 & \\
\hline & 4 & 85 & 12.31 & & & & 2 & & & 99 & & & & 3.85 & 24.62 & \\
\hline & & 5.56 & 19.38 & & & & & & & & 18. & & & 4.32 & 16.67 & \\
\hline & & & & & & & 29.79 & & & & & & & 0. & 33 & \\
\hline & & & 20 & & & & & & & & . & & & 48 & & \\
\hline & & .00 & 48.40 & & & & & & & & & & & 31.00 & 42.00 & \\
\hline & 92.06 & 7.78 & 81.11 & & & 44 & 57.78 & & & 16.67 & & & 87 & 44.44 & 61.11 & \\
\hline & & 6.36 & 45.12 & & & & & & & & & & & 22.48 & 36.90 & \\
\hline & & 6.21 & & & & 17.48 & 23 & & & & & & & 17.48 & 24.08 & \\
\hline & 100.00 & 100.00 & 100.00 & 00 & 100.00 & 100.00 & 100.00 & 10 & 67 & 80.00 & 96.00 & & 100.00 & 100.00 & 100.00 & 100.00 \\
\hline & & 21.74 & 26.52 & & & 15.22 & 20.87 & & & 19.57 & 23. & & .33 & 17.39 & 22.61 & \\
\hline & & & & & & & & & & & & & & & & \\
\hline & & 80.00 & 80.00 & & & 80.00 & 80.00 & & & & 80 & & & 30.00 & 80.00 & \\
\hline & 96.93 & 37.93 & 43.45 & & 77 & 24.83 & 40.00 & & 93 & 15.86 & 24.00 & & 76 & 24.83 & 39.59 & 34 \\
\hline $\mathrm{ND}$ & 95.85 & 27.22 & 44.81 & & 67 & 44.94 & 54.56 & & & 53.80 & 61.52 & & 94.79 & 44.30 & 55.57 & \\
\hline 10 & 875 & s. & 31.485 & 2.605 & 140 & 02 & 0 & 3.82 & 90.89 & 1 & 4 & 2406 & 8 & 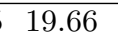 & 3.09 & 4150 \\
\hline
\end{tabular}


Table S10: Peptide dissimilarity between train (IEDB) and test (Japanese) datasets. Sequence identity column shows average sequence identity between test peptide sequence and most similar peptide sequence in the training dataset.

\begin{tabular}{ccc}
\hline Train dataset & Test dataset & Sequence identity \\
\hline \hline A0201-IEDB & A0201-Japanese & $55.2 \%$ \\
A0206-IEDB & A0206-Japanese & $46.8 \%$ \\
A2402-IEDB & A24020-Japanese & $51.1 \%$ \\
\hline
\end{tabular}

\section{Naturally-processed peptide prediction}

To train our models, we used the data from Machine Learning in Immunology competition (MLI-II) http://bio.dfci.harvard.edu/DFRMLI/HTML/natural. php. For each of the MHC-I alleles, a set of 8- to 11-mer peptides is given for training and testing. We directly train our models to recognize naturally processed and presented (NP) peptides, using "eluted" peptides as a positive set, and all other peptides (non-binders + non-eluted binders) as a negative set. We then test our models on the data composed of non-eluted binding peptides, non-binding peptides, and naturally processed ("eluted") peptides. We used the same training and test split as specified in the competition. In Table S11. we compare our approach hkSVM with the popular NetMHC method, which was used as a benchmark in the competition, as well as the recently introduced MHC-NP (Gigure et al., 2013) method that yielded state-of-the-art accuracy for naturally processed (NP) peptide prediction.

Table S11: Prediction of naturally processed and presented (NP) peptides (MLIII competition). Comparison of AUC scores.

\begin{tabular}{lcccc}
\hline & MHC-NP (E vs B+N) $^{\dagger}$ & MHC-NP $^{\dagger}$ & NetMHC $^{\dagger}$ & Our method \\
Allele & AUC & AUC & AUC & AUC \\
\hline \hline B0702 & 91.57 & 92.36 & 90.42 & $\mathbf{9 3 . 4 5}$ \\
B3501 & 91.87 & $\mathbf{9 3 . 6 7}$ & 90.9 & $\mathbf{9 3 . 5 4}$ \\
B4403 & 81.78 & 79.47 & 81.04 & $\mathbf{9 0 . 3 5}$ \\
B5301 & 84.01 & 85.15 & 66.51 & $\mathbf{9 1 . 1 4}$ \\
B5701 & 80.17 & $\mathbf{8 2 . 5 8}$ & 81.81 & $\mathbf{8 2 . 9 3}$ \\
\hline
\end{tabular}

${ }^{\dagger}$ quoted from (Gigure et al. 2013

\section{References}

Buus, S., Lauemøller, S., Worning, P., Kesmir, C., Frimurer, T., Corbet, S., Fomsgaard, A., Hilden, J., Holm, A., and Brunak, S. (2003). Sensitive quantitative predictions of peptide-MHC binding by a 'Query by Committee' artificial neural network approach. Tissue Antigens, 62(5), 378-384. 
Gigure, S., Drouin, A., Lacoste, A., Marchand, M., Corbeil, J., and Laviolette, F. (2013). MHCNP: Predicting peptides naturally processed by the MHC. Journal of Immunological Methods, 400401(0), $30-36$.

Kim, Y., Sidney, J., Buus, S., Sette, A., Nielsen, M., and Peters, B. (2014). Dataset size and composition impact the reliability of performance benchmarks for peptide-mhc binding predictions. $B M C$ Bioinformatics, 15(1), 241.

Lundegaard, C., Lund, O., and Nielsen, M. (2011). Prediction of epitopes using neural network based methods. Journal of Immunological Methods, 374(1-2), 26 - 34. High-throughput methods for immunology: Machine learning and automation.

Nielsen, M., Lundegaard, C., Worning, P., Lauemøller, S. L., Lamberth, K., Buus, S., Brunak, S., and Lund, O. (2003). Reliable prediction of T-cell epitopes using neural networks with novel sequence representations. Protein Science, 12(5), 1007-1017. 